\title{
Limb graft occlusion following endovascular aortic repair: Incidence, causes, treatment and prevention in a study cohort
}

\author{
GUOQUAN WANG $^{1}$, SHUITING ZHAI ${ }^{1}$, TIANXIAO LI ${ }^{1}$, XUAN LI $^{2}$, DANGHUI LU $^{1}$, BO WANG ${ }^{1}$, \\ DONGBIN ZHANG ${ }^{1}$, SHUAITAO SHI ${ }^{1}$, ZHIDONG ZHANG ${ }^{1}$, KAI LIANG ${ }^{1}$, \\ KEWEI ZHANG ${ }^{1}$, XIAOYANG FU ${ }^{1}, \mathrm{KUN} \mathrm{LI}^{1}$ and WEIXIAO LI ${ }^{1}$ \\ ${ }^{1}$ Department of Vascular and Endovascular Surgery, Henan Provincial Peoples' Hospital, Zhengzhou University, \\ Zhengzhou, Henan 450003; ${ }^{2}$ Department of Vascular and Endovascular Surgery, \\ Peking University Third Hospital, Beijing 100191, P.R. China
}

Received March 16, 2016; Accepted February 24, 2017

DOI: $10.3892 /$ etm.2017.4658

\begin{abstract}
The present study investigated the incidence, causes, treatment and prevention of limb graft occlusion following endovascular aortic repair (EVAR). A total of 66 cases of abdominal aortic aneurysm receiving EVAR at our department from January 2005 to December 2013 were enrolled. After EVAR, patients received routine antiplatelet therapy of $75 \mathrm{mg}$ PLAVIX for 6 months and then $100 \mathrm{mg}$ Aspirin for another 6 months by oral administration. According to previous clinical experiences, antiplatelet therapy is able to effectively reduce the incidence of iliac occlusion after EVAR. A total of 61 bifurcated grafts and 5 aortauniilac grafts $(127$ limbs in total) were used. Physical examination, ankle-brachial-index and computer tomographic angiography were performed at 10 days, at 3, 6 and 12 months and annually thereafter. It was found that $7 \mathrm{limbs}$ in 7 patients $(10.6 \%$ of patients, $5.5 \%$ of limbs) were occluded between 20 days and 12 months (average, $7.8 \pm 5.3$ months) after EVAR. Acute and severe ischemia was found in 2 cases, claudication was in found 3 cases, asthenia in both legs was found in 1 case and 1 case was asymptomatic. Femoral-femoral bypass, femoral-femoral bypass and stenting, aorto-iliac/femoral bypass, thrombectomy and conservative treatment were performed in 1 patient each and thrombectomy together with stenting was performed in 2 cases. Limb graft occlusion was not rare after EVAR. Treatment of this complication included surgery and endovascular therapy such as bypass, thrombectomy and thrombolysis. In conclusion, aggressive pre-emptive treatment including angioplasty and stenting prevented occlusion in certain cases.
\end{abstract}

Correspondence to: Dr Shuiting Zhai, Department of Vascular and Endovascular Surgery, Henan Provincial Peoples' Hospital, Zhengzhou University, 7 Weiwu Road, Zhengzhou, Henan 450003, P.R. China

E-mail: 15713662288@163.com

Key words: endovascular repair, abdominal aortic aneurysm, limb graft occlusion, endovascular aortic repair

\section{Introduction}

Abdominal aortic aneurysm (AAA) is defined as a permanent and irreversible dilation of the abdominal aorta (1). Age, male sex, personal history of atherosclerotic cardiovascular disease, smoking and hypertension are all associated with the occurrence of AAA. AAA accounts for $\sim 15,000$ deaths in the USA per year (2). In addition, $30-40 \%$ of patients with ruptured AAA die without surgery (3-5). The operative mortality rate of AAA is $40-50 \%$ (3-6), however, the overall mortality of AAA rupture is $80-90 \%$.

Since the first stent graft system designed for endovascular aortic repair (EVAR) of AAA was approved by the US Food and Drug Administration in 1999, EVAR has been a major treatment for AAA (6). Compared to traditional open surgery, EVAR has advantages, including lower blood loss, less pain, shorter hospitalization, more rapid recovery as well as lower morbidity and mortality (7). Its advantage is obvious, particularly in patients with early-stage AAA. However, complications such as rupture, endoleak, device migration and limb graft occlusion are usually found after EVAR $(2-4,7,8)$. In addition, patients receiving EVAR have been reported to have high rates of re-intervention (8).

Limb graft occlusion is not rare and represents a serious complication of EVAR. It is the third most common reason for readmission after EVAR. The present study retrospectively evaluated the incidence, causes, and methods of treatment and prevention of limb graft occlusion following EVAR encountered at our department.

\section{Patients and methods}

Patient data. A total of 66 patients who underwent EVAR at the Department of Vascular and Endovascular Surgery (Henan Provincial Peoples' Hospital, Zhengzhou, China) from January 2005 to December 2013 were enrolled in the present study. Clinical data, including computer tomographic angiography (CTA) data, digital subtraction angiography (DSA) data as well as information on operative management and outcome were collected and retrospectively analyzed. Based on CTA, the standards of EVAR were as follows: i) An 
Table I. General information on the 7 cases of limb graft occlusion following EVAR.

\begin{tabular}{llcclll}
\hline Case no. & Sex & Age (years) & Aneurysm & Graft company & Graft type & Time of occlusion after EVAR \\
\hline 1 & Male & 64 & $\mathrm{~T}$ & Medtronic & Bifurcated & 3 months \\
2 & Male & 62 & $\mathrm{~T}$ & Medtronic & Bifurcated & 12 months \\
3 & Male & 77 & $\mathrm{~T}$ & Medtronic & Bifurcated & 3 months \\
4 & Male & 29 & $\mathrm{~F}$ & Medtronic & Bifurcated & 12 months \\
5 & Male & 76 & $\mathrm{~T}$ & Medtronic & AUI & 20 days \\
6 & Male & 43 & $\mathrm{~T}$ & Cook & Bifurcated & 12 months \\
7 & Female & 80 & $\mathrm{~T}$ & Cook & Bifurcated & 12 months \\
\hline
\end{tabular}

T, true aneurysm; F, false aneurysm; AUI, aortauniilac; EVAR, endovascular aortic repair.

aneurysm diameter of $>50 \mathrm{~mm}$; ii) an aneurysm diameter of $<50 \mathrm{~mm}$ but fulfillment of the following characteristics: a) An increase in the aneurysm diameter of $>5 \mathrm{~mm}$ over the last year; b) irregular shape of the aneurysm associated with a high risk of rupture; c) abdominal discomfort associated with the aneurysm; d) large amounts of thrombosis in the aneurysm with a high risk of embolism. In total, 61 bifurcated grafts and 5 aortauniilac (AUI) grafts (127 limbs in total) were assessed. Prior written and informed consent was obtained from each patient and the study was approved by the Ethics Review Board of Henan Provincial Peoples' Hospital (Zhengzhou, China).

Grafts. Talent, endurant and AUI grafts were purchased from Medtronic (Medtronic Inc., Minneapolis, MN, USA). The Zenith graft was from Cook Medical Inc. (Bloomington, IN, USA). The Wallstent graft was from Boston Scientific Corp. (Boston, MA, USA).

EVAR treatment. After general anesthesia and bilateral inguinal oblique incision, small and sub-centimeter incisions were made over the femoral artery. Placement of a $6 \mathrm{~F}$ femoral artery sheath was performed. The $5 \mathrm{~F}$ gold-labeled pigtail catheter was inserted into the femoral artery, which was connected with a high-pressure syringe and ventilator. Systemic heparinization was performed. A 4F hunter catheter was then inserted and a Cook Lunderquist guide wire (Cook Medical Inc.) was inserted into the femoral artery. A bracket was guided by the wire and once the bracket was completely released, the conveying system was carefully recycled into the sheath and retreated to the bracket. A high-pressure syringe was then connected and aortography was performed to compare the conditions with those before stent implantation. The delivery sheath was pulled out. Medtronic compliant balloon (AB46) expansion of the stent ends and the interface part of the exit site was performed. Angiography was performed once again when the bracket was in a good position and state without obvious internal leakage. After delivery, the guide wire and sheath were removed and the wound was closed. Subsequent to surgery, the general condition of the patients was monitored.

Post-operative treatment and follow-up. Antiplatelet treatment was performed in all of the 66 patients during routine review after EVAR and heparin was used in the case of thrombosis.
The antiplatelet treatment was performed for 12 months by oral administration of $75 \mathrm{mg}$ PLAVIX (Sanofi Winthrop S.A., Paris, France) daily for 6 months and 100 mg Aspirin (Bayer, Leverkusen, Germany) daily for another 6 months. At 10 days, 3, 6 and 12 months and annually thereafter, duplex ultrasonography or CTA was performed. Readmission was suggested if the patient complained of any discomfort, particularly when abdominal or limb symptoms appeared. Review examinations, including physical examination, ankle-brachial index, CTA and if necessary DSA were performed in patients with limb ischemia.

Statistical analysis. All statistical analyses were performed using SPSS version 17.0 (SPSS, Inc., Chicago, IL, USA) for Windows. Values are expressed as the mean \pm standard deviation. An independent-samples t-test was performed for comparison between the two groups. $\mathrm{P}<0.05$ was considered to indicate a statistically significant difference.

\section{Results}

Incidence of limb graft occlusion following EVAR. To analyze the incidence of limb graft occlusion following EVAR, the patients were followed up. As shown in Tables I and II, a total of 7 limbs in 7 patients were occluded between 20 days and 12 months (average, 7.8 \pm 5.3 months) after EVAR. The total incidence was $10.6 \%(7 / 66)$ in the patients and $5.5 \%(7 / 127)$ in the limbs. Among the 7 patients, 6 bifurcated grafts and 1 AUI graft were used and 5 right-limb grafts and 2 left-limb grafts were applied (Tables I and II). For Patients with limb graft occlusion, the follow-up time ranged from 1-38 months, with an average follow-up time of $24.2 \pm 16.6$ months. The time of the appearance of limb occlusion showed a great variation, with the shortest being 20 days in 1 case, 12 months in 4 cases and 3 months in 2 cases. The clinical manifestation also varied within the cohort; one case was asymptomatic, acute and severe ischemia was found in 2 cases, claudication was found in 3 cases and asthenia was found in 1 case. Taken together, these results showed that limb graft occlusion following EVAR was not rare.

Causes of limb graft occlusion following EVAR. To investigate the causes of limb graft occlusion following EVAR, clinical data of each patient were analyzed. Anatomical factors were 


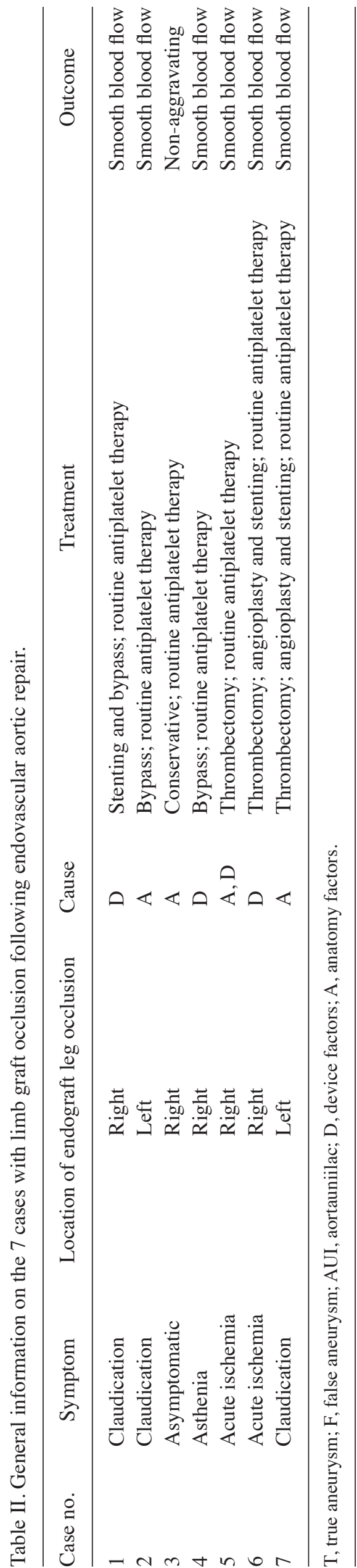

found in 3 patients. One case was a 77-year-old man with no symptoms. Regular examination was performed at 3 months after EVAR and the pulse of the right femoral and popliteal arteries was weak. CTA indicated that the right leg graft was occluded. Another case was a 62-year-old man with left leg graft occlusion occurring at 12 months after EVAR. The other case was a 80-year-old female whose left leg graft was occluded because of calcification and tortuousness of the iliac artery. This occlusion was also found at 12 months after EVAR.

Device factors were found in 3 patients. One was a 29-year-old man who had a false aneurysm due to trauma. Due to the small diameter of the abdominal aorta, a bifurcated stent graft was unsuitable for him and 'AUI and femoral-femoral bypass' was performed instead. At 6 months after EVAR, he complained of asthenia in both legs; however, no obvious signs were detected by ABI or CTA. At 12 months after EVAR, the symptoms had not aggravated, but CTA examination indicated that AUI and artificial graft were filled with thrombus. Another case was a 43-year-old man (Figs. 1-4). Aorta CTA confirms the formation of abdominal aortic pseudoaneurysm (Fig. 1A). After detailed evaluation, EVAR surgery was performed. At 10 days after endovascular aortic repair, the pseudoaneurysm was observed to be completed eradicated (Fig. 1B). After 12 months, the right lower leg was cold with limited activity. The emergency CTA suggested occlusion in the right iliac artery (Fig. 2A). The MIP indicated a right iliac artery occlusion (Fig. 2B). Combined balloon dilatation and stent implantation was conducted. At 10 days after reoperation, blood flow in the right iliac artery was restored (Fig. 3A) and stent grafts were well positioned and well shaped (Fig. 3B). The patient was examined by CTA 16 weeks after reoperation. The right iliac artery was restored (Fig. 4A) and stent grafts were well positioned and well shaped (Fig. 4B). The right leg was occluded at 12 months after EVAR. DSA revealed a fibro cap at the exit of the stent graft. Meanwhile, the right leg graft partly covered the left leg graft and affected the blood flow of the left leg.

Occlusion due to anatomic as well as device factors was found in a 76-year-old man. The right leg graft extended to the external iliac artery and he suffered acute and severe ischemia in the right leg at 20 days after EVAR. Symptoms of pallor, pain and weak pulse were present in the right leg, whereas the left leg was normal. An urgent angiography revealed that there was no blood flow in the right leg graft. In summary, the causes of limb graft occlusion following EVAR included anatomic factors, device factors or their combination.

Treatment of limb graft occlusion following EVAR. To identify the effects of various treatment methods for limb graft occlusion following EVAR, the methods used and the prognosis of the patients were analyzed. The thrombectomy, aorto-right common iliac/left femoral artery bifurcated artificial graft bypass, femoral-femoral artificial graft bypass, femoral-femoral artery bypass and stent, and conservative treatment were used in 1 case and thrombectomy together with a stent was used in 2 cases. All patients except for the patient who received conservative treatment had a good outcome. None of the patients died and no amputation due to limb graft occlusion was required. In conclusion, the results 
A

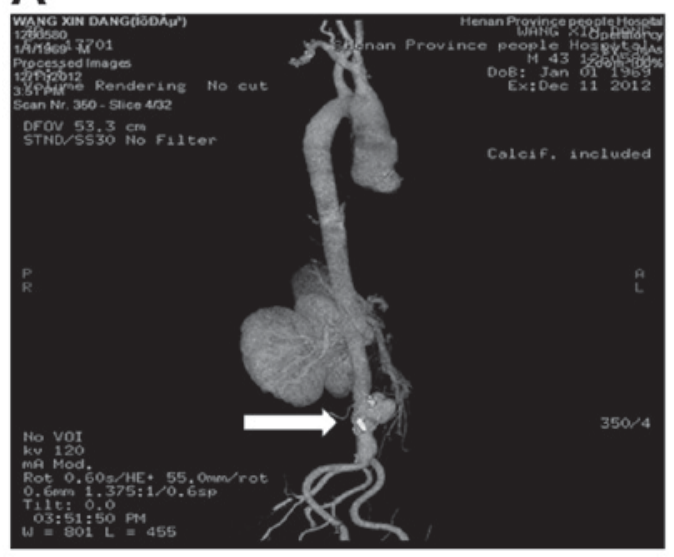

B

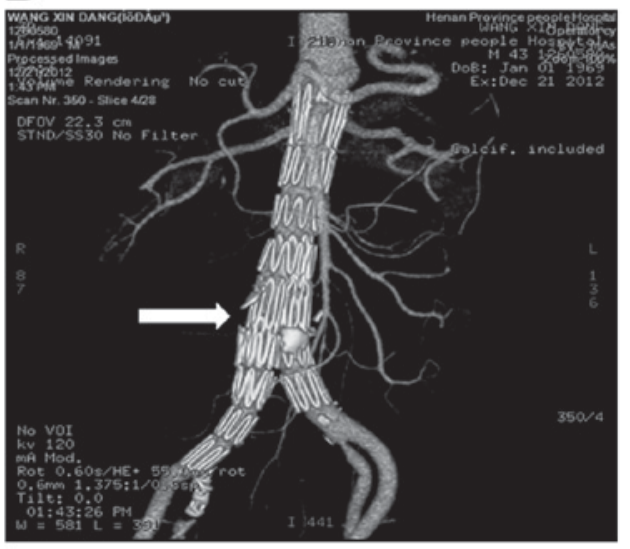

Figure 1. Three-dimensional reconstruction of 64-slice computer tomographic angiography images of the 43-year-old patient (case no. 6). (A) A false aneurysm on the lower part of the aorta ventralis is shown (arrow). (B) At 10 days after endovascular aortic repair, the false aneurysm disappeared completely (arrow).

A

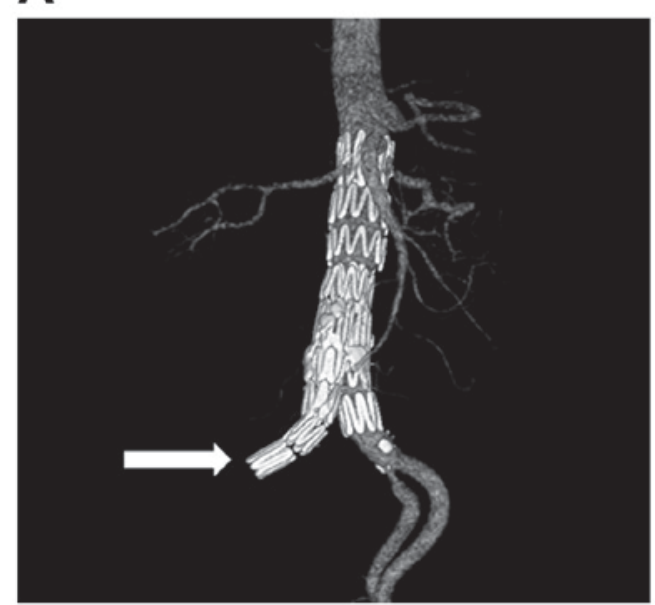

B

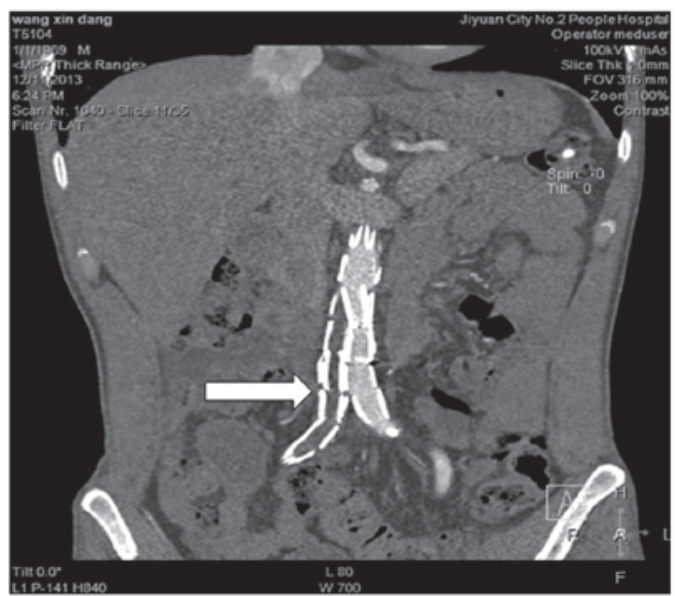

Figure 2. Three-dimensional reconstruction of 64-slice computer tomographic angiography images of the 43-year-old patient (case no. 6) at 1 year after operation showing (A) the artery and (B) the full body. Occlusion in the right iliac artery was observed (arrows). A low-density shadow was visible, indicating the formation of thrombosis.

A

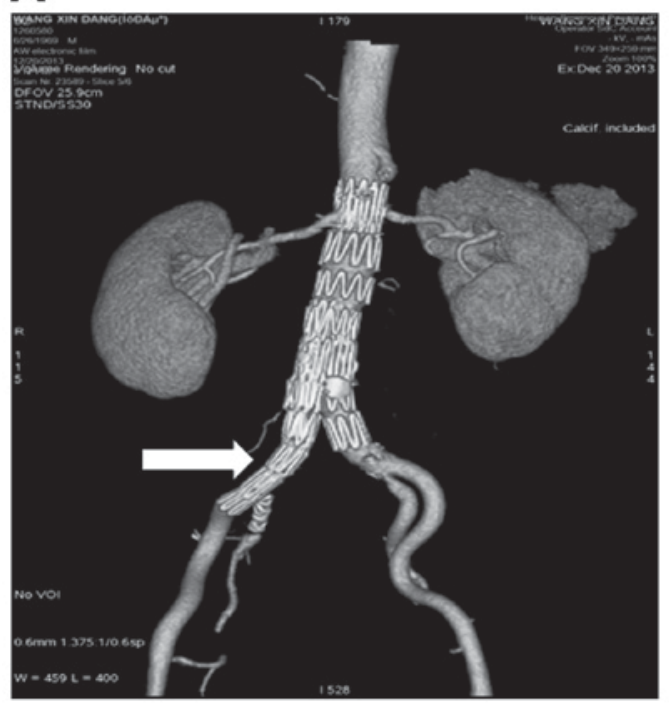

B

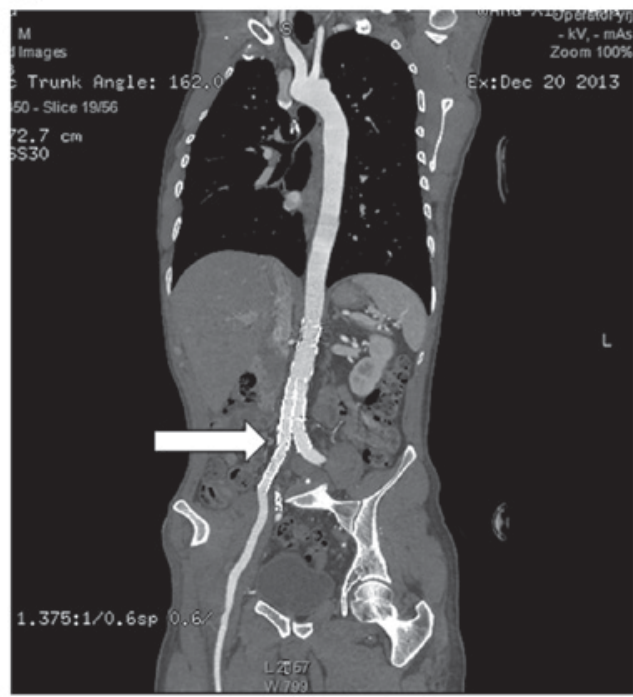

Figure 3. Three-dimensional reconstruction of 64-slice computer tomographic angiography images of the 43-year-old patient (case no. 6) at 10 days after reoperation showing (A) the artery and (B) the full body. Blood flow in the right iliac artery was restored and the development was good (arrows). 
A

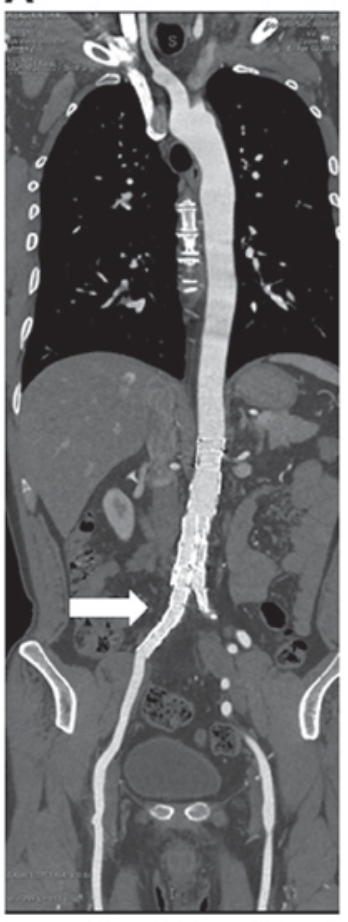

B

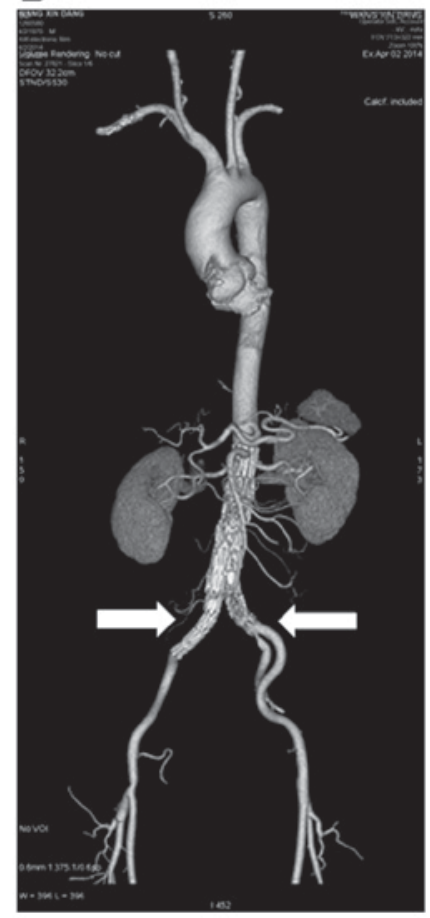

Figure 4. Three-dimensional reconstruction of 64-slice computer tomographic angiography images of the 43 -year-old patient (case no. 6) at 16 weeks after reoperation showing (A) the artery and (B) the full body. Blood flow in the right iliac artery was restored and the development was good (arrows).

indicated that with appropriate treatment, limb graft occlusion following EVAR has a favorable prognosis.

\section{Discussion}

Limb graft occlusion is not a rare but serious complication following EVAR. Woody and Makaroun (9) reported that it is the third most common reason for readmission after EVAR. Sivamurthy et al (10) retrospectively reviewed 248 patients who underwent EVAR from 1999 to 2004, including 13 instances of limb thrombosis in 13 patients with a total incidence of $5.2 \%$ in patients and $2.7 \%$ in limbs. Cochennec et al (11) reviewed 460 AAA patients who were selectively treated with a variety of commercially available stent grafts from 1995 to 2005 . They used 369 bifurcated and 91 AUI grafts (829 limbs) in their study and found that 36 limbs in 33 patients (in $7.2 \%$ of patients and $4.3 \%$ of limbs) were occluded. The total incidence in the present study was $10.6 \%$ in patients and $5.5 \%$ in limbs, which was consistent with the results of previous studies.

Limb graft occlusion following EVAR is a disease caused by numerous factors, which may be divided into 3 major categories. The first is anatomic factors, the second is device (graft) factors and the final one contains the two aspects combined. Anatomic factors includes small artery size (particularly for female patients), aneurysm angulation of $\geq 60^{\circ}$, calcified and narrowed aortic bifurcation, tortuous iliac artery and iliac artery dissection. According to Woody and Makaroun (9), these anatomic characteristics led to kinking of the iliac limb of the endograft. Extrinsic compression of the endograft due to a significant thrombus load within the aneurysm sac may lead to stenosis of the proximal portion of the endograft limb.
Likewise, a narrow aortic bifurcation may compress the iliac graft limbs. In the present study 3 limbs in 3 patients were occluded due to anatomic factors.

Carpenter et al (12) reported that each case of limb thrombosis in their study was due to a mechanical cause. Device factors included graft migration, insufficient graft size and low radial force. Graft migration is a rare but serious complication, and in the present study, a 64-year-old man who underwent EVAR 4 months previously was diagnosed with a ruptured AAA. During the operation, it was found that the limb graft was incomplete and another type of graft (Fluency; Bard Peripheral Vascular, Inc., Tempe, AZ, USA) was selected for him. One month after EVAR, CTA showed that the graft was fluent; however, 3 month after EVAR, claudication was found in his right leg. CTA indicated that the right limb graft was occluded. During the operation, it was found that the wire was not able to pass through the occluded graft and that the limb graft had migrated from the main body. As a result, a femoral-femoral artificial bypass was performed. Grafts lack sufficient support with a weak radial force being a particularly significant cause and kinking is common under these circumstances. Previous studies have also described this problem (13). Excessive oversizing of the graft may lead to infolding of the graft material within the graft lumen $(14,15)$.

Finally, twisting of the graft limbs may occur. This is associated with anatomic factors as well as with characteristics of the endograft delivery system. Twisting of grafted limbs narrows the lumen and predisposes to thrombosis. It has been reported that a graft extending into the external iliac artery is a high risk factor for occlusion (16). When the graft extends into the external iliac artery, the likelihood of kinking is higher. Under these circumstances, the blood flow is decreased.

Treatment can be divided into 4 aspects: Endovascular, surgery, hybrid and conservative treatment. Certain clinicians prefer endovascular treatment, insist that thrombosis preserves the original shape of grafts and successfully implement angiography or stenting (17). However, artificial graft bypass is considered the first choice of treatment for others. One important reason for the preference of extra-anatomic bypass is that revascularization may prevent embolization of the hypogastric artery secondary to thrombectomy or thrombolysis. According to our experience, thrombolysis or thrombectomy with adjunctive stenting is an effective treatment method for limb graft occlusion, as thrombectomy combined with stent implantation effectively addresses iliac artery stenosis caused by stent displacement.

The best treatment method for limb graft occlusion is prevention. It is better to select an individual therapeutic schedule and an appropriate graft for individual patients at the preoperative stage. According to previous clinical experiences, antiplatelet therapy was observed to effectively reduce the incidence of iliac occlusion after EVAR. Therefore, antiplatelet therapy was performed in all patients of the current study for 12 months. For female patients or those with a small aortic size, open surgery may be more suitable if no appropriate stent-graft is available. The modalities of the operation are undoubtedly key to preventing limb graft occlusion. During the final steps of the operation, balloon dilation may be re-performed if angiography reveals that the graft shows kinking or twisting. If the shape and blood flow remain 
unsatisfactory, stenting is necessary. Sivamurthy et al (10) used adjunctive primary stenting during EVAR of lower limb graft occlusion, which was efficient and safe. Kissing balloon during angiography is another technique for preventing a previously normal contralateral limb graft from being compromised. However, single-plane angiography is inadequate for angiography. Multiplanar angiography provides further details and may reveal limb problems. Intra-vascular ultrasonography may provide valuable information, as it may detect graft redundancy and the resultant infolding of graft fabric into the lumen that is frequently undetected by angiography. At present, EVAR is followed up at 1,6 and 12 months, and yearly thereafter. Sivamurthy et al (10) reported that most occlusions occur within 6 months after endovascular AAA repair. Thus, the current routine follow-up regimen appears to only have a small impact in the prevention of limb thrombosis. In most cases, graft thrombosis occurs within 6 months after stent-graft insertion and the patients only undergo one routine imaging at 1 month. In the present study, $60 \%$ of occlusions occurred within 6 months and a different follow-up schedule of 10 days, 3, 6 and 12 months and once annually thereafter was employed. Furthermore, clinical experience of authors of the present study suggests that routine antiplatelet treatment after EVAR is a good preventive measure, particularly for patients with peripheral artery disease.

\section{References}

1. Scali ST, Feezor RJ, Huber TS and Beck AW: Acute bilateral renal artery chimney stent thrombosis after endovascular repair of a juxtarenal abdominal aortic aneurysm. J Vasc Surg 61: 1058-1061, 2015.

2. Lim S, Halandras PM,Park T,Lee Y,CrisostomoP,Hershberger R, Aulivola B and Cho JS: Outcomes of endovascular abdominal aortic aneurysm repair in high-risk patients. J Vasc Surg 61: 862-868, 2015.

3. Wang Y, Joannic D, Delassus P, Lalande A, Juillion P and Fontaine JF: Comparison of the strain field of abdominal aortic aneurysm measured by magnetic resonance imaging and stereovision: A feasibility study for prediction of the risk of rupture of aortic abdominal aneurysm. J Biomech 48: 1158-1164, 2015.

4. Törnqvist P, Dias N, Sonesson B, Kristmundsson T and Resch T: Intra-operative cone beam computed tomography can help avoid reinterventions and reduce CT follow up after infrarenal EVAR. Eur J Vasc Endovasc Surg 49: 390-395, 2015.
5. Troisi $\mathrm{N}$ and Torsello G: Commentary: New-generation devices and adjunctive procedures are the key elements to expanding the indications for endovascular aneurysm repair. J Endovase Ther 22: 179-181, 2015.

6. You JH, Park CB, Park HK, Jin ES and Kim CJ: Endovascular repair of graft limb occlusion after endovascular repair for abdominal aortic aneurysm using 0.014 -inch guidewire and coronary balloon. Int J Cardiol 153: e37-e38, 2011.

7. Alder L, Al-Jarrah Q, Rahi MA, Wilde N and Al-Khaffaf H: Percutaneous catheter-directed thrombolysis for treatment of complete body and bilateral limb endovascular aortic graft occlusion. EJVES Extra 24: e37-e38, 2012.

8. Liaw JV, Clark M, Gibbs R, Jenkins M, Cheshire N and Hamady M: Update: Complications and management of infrarenal EVAR. Eur J Radiol 71: 541-551, 2009.

9. Woody JD and Makaroun MS: Endovascular graft limb occlusion. Semin Vasc Surg 17: 262-267, 2004.

10. Sivamurthy N, Schneider DB, Reilly LM, Rapp JH, Skovobogatyy $\mathrm{H}$ and Chuter TA: Adjunctive primary stenting of Zenith endograft limbs during endovascular abdominal aortic aneurysm repair: Implications for limb patency. J Vasc Surg 43: 662-670, 2006.

11. Cochennec F, Becquemin JP, Desgranges P, Allaire E, Kobeiter H and Roudot-Thoraval F: Limb graft occlusion following EVAR: Clinical pattern, outcomes and predictive factors of occurrence. Eur J Vasc Endovasc Surg 34: 59-65, 2007.

12. Carpenter JP, Anderson WN, Brewster DC, Kwolek C, Makaroun M, Martin J, McCann R, McKinsey J and Beebe HG; Lifepath Investigators: Multicenter pivotal trial results of the Lifepath System for endovascular aortic aneurysm repair. J Vasc Surg 39: 34-43, 2004

13. Erzurum VZ, Sampram ES, Sarac TP, Lyden SP, Clair DG, Greenberg RK, O'Hara PJ, Kashyap VS and Ouriel K: Initial management and outcome of aortic endograft limb occlusion. J Vasc Surg 40: 419-423, 2004.

14. Becquemin JP, Kelley L, Zubilewicz T, Desgranges P, Lapeyre M and Kobeiter H: Outcomes of secondary interventions after abdominal aortic aneurysm endovascular repair. J Vasc Surg 39: 298-305, 2004

15. Wales L, Dunckley M, Bohm N, Kwok T, Bratby M, Morgan R, Thompson M and Loftus I: Device-specific outcomes following endovascular aortic aneurysm repair. Eur J Vasc Endovasc Surg 36: 661-667, 2008

16. Maldonado TS, Rockman CB, Riles E, Douglas D, Adelman MA, Jacobowitz GR, Gagne PJ, Nalbandian MN, Cayne NS, Lamparello PJ, et al: Ischemic complications after endovascular abdominal aortic aneurysm repair. J Vasc Surg 40: 703-710, 2004.

17. Becquemin JP, Allaire E, Desgranges P and Kobeiter H: Delayed complications following EVAR. Tech Vasc Interv Radiol 8: 30-40, 2005 